\title{
KINEMATICAL CONSTRAINTS OF HELICITY AMPLITUDES
}

\author{
Donald M. LEVINE * \\ Department of Physics, University of Michigan, \\ Ann Arbor, Michigan 48104
}

Received 24 April 1969

\begin{abstract}
The kinematical constraints of helicity amplitudes are studied using the general ideas of Jackson and Hite. The helicity amplitudes are expressed in terms of a Taylor series expansion from which all constraints at threshold and pseudothreshold can be trivially found. The simplicity of this approach is illustrated by deriving all of the constraints for $\pi \rho \rightarrow \mathrm{N} \overline{\mathrm{N}}$ and $\pi \rho \rightarrow \Delta \overline{\mathrm{N}}$.
\end{abstract}

\section{INTRODUCTION}

One of the difficulties in developing dynamical theories for the description of scattering processes has been the determination of which characteristics of the processes are purely kinematical and which are purely dynamical. The extraction of all of the kinematical aspects of the scattering processes $1+2 \rightarrow 3+4$ from helicity amplitudes [1] has been attempted by several authors using many different approaches [2-5]. One of the more interesting methods is that of Jackson and Hite [3] who use non-relativistic ideas coupled with very elementary mathematics to determine all of the factorizable kinematical singularities in the $s$ (c.m. energy squared) plane (they indicated how one might determine the singularities in the $w=\sqrt{s}$ plane but they did not actually carry this out; this was done by Henyey [3]). While Jackson and Hite outline a general approach to solving the problem of finding the kinematical constraints, they do not actually carry out the mathematics except in one or two specific cases. This paper shows that the basic ideas used by Jackson and Hite to find the factorizable kinematical singularities can also be used to solve the problem of finding all kinematical constraints. We are able to show that the helicity scattering amplitudes for $1+2 \rightarrow 3+4$ can be expressed in a Taylor series like expansion about each threshold and/or pseudothreshold and that all of the kinematical constraints can be extracted from this one simple equation. The simplicity of this equation is illustrated by studying the reasonably complicated processes $\rho \pi \rightarrow \mathrm{N} \overline{\mathrm{N}}$ and $\rho \pi \rightarrow \overline{\mathrm{N}} \Delta$.

Although Trueman [6], working independently, has already discussed the

* Work partially supported by the Atomic Energy Commission. 
problem of kinematical constraints from an entirely different point of view, the present paper affords a deeper insight into the physical origins of the constraints and shows explicitly that kinematical singularities and constraints are caused by the same phenomena. While Trueman uses different phase conventions and discusses helicity amplitudes rather than parity conserving helicity amplitudes as done here, the results of Trueman's work and this paper can be shown to be completely consistent with one another.

Sect. 2 summarizes the results of this paper and states a set of rules for finding all of the kinematical constraints. Sect. 3 gives examples of the use of these rules. In appendix A notations and conventions are discussed while the results of this paper are derived in detail in appendix B. Finally in appendix $\mathrm{C}$ we review the problem of finding the kinematical constraints at $s=0$ which exist independently of whether or not $m_{1}=m_{2}$ and/or $m_{3}=m_{4}$ and are not included in the results of sect. 2 .

\section{KINEMATICAL CONSTRAINTS}

As is well known the helicity scattering amplitudes for two body scattering processes $1+2 \rightarrow 3+4$ have kinematical singularities at the thresholds $\left( \pm\left(m_{i}+m_{j}\right)\right)$ and pseudothresholds $\left( \pm\left(m_{i}-m_{j}\right)\right)$ of the incoming and outgoing particles. By carefully investigating why these singularities occur, we have been able to explicitly separate the general scattering amplitude for twobody scattering into a sum of terms each having a different degree of singularity at threshold and/or pseudothreshold. After multiplying this sum by the appropriate factors to eliminate all threshold and pseudothreshold kinematical singularities, we essentially have a Taylor series about the thresholds and/or pseudothresholds. We can now use this series to trivially determine all of the kinematical constraints at the thresholds and/or pseudothresholds: for any given process at any threshold or pseudothreshold, $\tau$, (i.e. $\tau= \pm\left(m_{i} \pm m_{j}\right)$ ) we set the c.m. energy squared, $s$, (for the case BF $\rightarrow \mathrm{BF}$, use $\tau=\sqrt{s}$ where $\mathrm{B}$ represents a baryon and $\mathrm{F}$ a fermion) * equal to $\tau^{2}$ thus forcing all but the first terms in the sum for each scattering amplitude equal to zero. Since several of these first terms will be proportional, we have a series of constraints which are called non-derivative. Next divide the scattering amplitude by $\tau^{2}$ (use $\tau^{2}$ because of angular momentum conservation - see appendix B) and then set $s=\tau^{2}$ (or $\sqrt{s}=\tau$ for BF $\rightarrow \mathrm{BF}$ ) and thus all but the first two terms in the series go to zero. For many scattering processes the number of independent scattering amplitudes is still larger than the number of unknowns introduced in this expansion and thus there are more constraints; these are called first derivative constraints because they involve the second terms in a Taylor series. Next we divide the amplitudes by $\tau^{4}$ and then set $s=\tau^{2}$ (or $\sqrt{s}=\tau$ ) and determine if we have still more constraints (second derivative constraints). We can continue this process of first dividing by even powers of $\tau$ and then setting

* As indicated by ref. [3] one need only use the $\sqrt{s}$ plane for BF reactions while for all other reactions the use of $\sqrt{s}$ and $s$ planes give identical results (see eq. (12)). 
$s=\tau^{2}$ (or $\sqrt{s}=\tau$ ) until the number of unknown equals the number of independent scattering amplitudes, for then no additional constraints exist.

While the mathematics of developing this method is not difficult we shall omit it here (see appendix B for details) and simply state the results. The Taylor series like expansion mentioned above for the $s$ (c.m. energy) channel parity conserving helicity amplitude free of kinematical singularities for the process $1+2 \rightarrow 3+4$ (see fig. 1) is:

$\tilde{f}_{\lambda_{3} \lambda_{4} ; \lambda_{1} \lambda_{2}}^{\eta}(s, t)$

$$
\begin{aligned}
& =\beta \sum_{D} \sum_{S_{i j}=S_{i}+S_{j}}^{S_{i}+S_{j}-D^{-} \alpha_{\eta}} \frac{\left\langle S_{i} \lambda_{i} S_{j}-\lambda_{j} \mid S_{i j} \lambda_{i}-\lambda_{j}\right\rangle}{\left(\left(S_{i j}+\lambda_{i}-\lambda_{j}\right) !\left(S_{i j}+\lambda_{j}-\lambda_{i}\right) !\right)^{\frac{1}{2}}}\left(\lambda_{i}-\lambda_{j}\right)^{D+\alpha_{\eta}-\xi} \\
& \times g_{S_{i j}}^{D, \eta}\left(\lambda_{a} b S_{a b} S_{i j} L_{a b^{J}}\right)\left(\sqrt{s}-\tau_{i j}\right)^{\frac{1}{2} D},
\end{aligned}
$$

where:

$$
\begin{aligned}
& \lambda=\lambda_{1}-\lambda_{2}, \quad \mu=\lambda_{3}-\lambda_{4}, \quad S_{i} \equiv \text { spin of } i \text { th particle }, \\
& \eta=(-)^{J} P(J=\text { total angular momentum, } P=\text { parity }) \text {, } \\
& \xi=S_{i j}-\left(S_{i}+S_{j}\right) \\
& \beta=\text { phase factor }=\left\{\begin{array}{lll}
\mathrm{e}^{i \pi\left(\lambda_{3}-\lambda_{4}\right)} & \text { for } & i, j=3,4, \\
\mathrm{e}^{-i \pi\left(\lambda_{1}-\lambda_{2}\right)} & \text { for } & i, j=1,2 .
\end{array}\right. \\
& D=0,2,4,6, \ldots \text {, } \\
& \tau_{i j}=\text { symbol for any one of the four possible thresholds or pseudo- }
\end{aligned}
$$
$\begin{aligned} g_{s i j}^{D, \eta}= & \text { the } i \text { th term in the expansion and is a function of its arguments } \\ & \text { where } a, b \text { refer to the two particles whose threshold or pseu- }\end{aligned}$

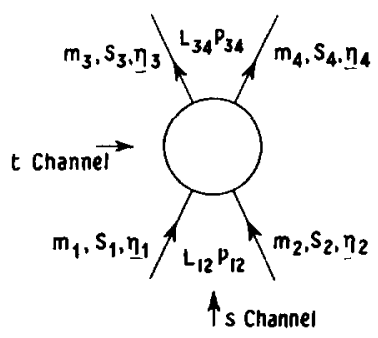

Fig. 1. The kinematics of $1+2 \rightarrow 3+4$ where: $m, S, \lambda, \eta$ are mass, spin, helicity, intrinsic parity, $L_{12}\left(L_{34}\right)$ is total orbital angular momentum of incoming (outgoing) particles and $P_{12}\left(P_{34}\right)$ is center-of-mass linear momentum of incoming (outgoing) particles. 
dothreshold we are not studying and $L$ is the orbital angular momentum.

$\alpha_{\eta}=$ factor necessary to insure parity conservation. From ref. [3] we find $\left(\alpha_{\eta}=\alpha_{+}\right.$for $\eta=+$ and $\alpha_{\eta}=\alpha_{-}$for $\left.\eta=-\right)$

$$
\begin{aligned}
\alpha & =\frac{1}{2}\left(1-\eta \eta_{i} \eta_{j}(-1)^{S_{i}+S_{j}-v}\right), & \tau & =m_{i}+m_{j}, \\
& =\frac{1}{2}\left(1-\eta \eta_{i} \eta_{j}(-1)^{-S_{i}-S_{j}-v}\right), & \tau & =-\left(m_{i}+m_{j}\right), \\
& =\frac{1}{2}\left(1-\eta \eta_{i} \eta_{j}(-1)^{S_{i}-S_{j}-v}\right), & \tau & =m_{i}-m_{j}, \\
& =\frac{1}{2}\left(1-\eta \eta_{i} \eta_{j}(-1)^{-S_{i}+S_{j}-v}\right), & \tau & =-m_{i}+m_{j},
\end{aligned}
$$

for $v=0\left(\frac{1}{2}\right)$ if $S_{i}+S_{j}=$ integer (half integer).

As indicated above, eq. (1) does not determine the constraints which occur at $s=0$ independently of whether or not $m_{1}=m_{2}$ and/or $m_{3}=m_{4}$. Therefore the results contained in eqs. (1), (4) and (5) at pseudothreshold for $m_{i}=m_{j}$ are only valid when these $s=0$ constraints are excluded from consideration. In appendix $C$, we discuss the problem of finding these additional constraints. For completeness and clarity we list the result: these constraints at $s=0$ for each amplitude such that $\lambda, \mu \neq 0$ are:

$$
\begin{aligned}
\tilde{f}_{\lambda_{3} \lambda_{4} ; \lambda_{1} \lambda_{2}}^{+}(s, t) & =-\tilde{a} \frac{\lambda \mu}{|\lambda \cdot \mu|} \tilde{f}_{\lambda_{3} \lambda_{4} ; \lambda_{1} \lambda_{2}}^{-}, \\
\tilde{a} & = \pm \text { if }\left(m_{1}^{2}-m_{2}^{2}\right)\left(m_{3}^{2}-m_{4}^{2}\right)_{<0}^{>0} \text { all masses unequal } \\
& = \pm \text { if }\left(m_{a}^{2}-m_{b}^{2}\right)_{<0}^{>0}, \quad m_{i}=m_{j}, \quad m_{a} \neq m_{b}, \\
& =+ \text { if } m_{1}=m_{2}=m_{3}=m_{4} .
\end{aligned}
$$

Eq. (1) is extremely simple to use in order to find the kinematical constraints. Before illustrating this simplicity by studying the reasonably complicated process $\pi \rho \rightarrow \overrightarrow{\mathrm{N}} \Delta$, we list two equations (see appendix $\mathrm{B}$ for the derivation) which determine the number of constraints in any process and the number of terms (i.e., derivatives) that must be included in the expansion of eq. (1) in order to derive all of these constraints.

If $M_{+}\left(M_{-}\right)$is the number of independent amplitudes for $\eta=+(-)$ with $\lambda_{a}, \lambda_{b}$ fixed but unspecified, the number of derivatives $N_{ \pm}$which must be considered at the threshold or pseudothreshold $\tau_{i j}$ of particles $i, j$ in order to determine all of the constraints can be found by solving

$$
n\left(3+\alpha_{ \pm}\right)-2=\min \left(M_{ \pm}, S_{i}+S_{i}-v\right),
$$

for $n$ and then setting 
$N_{ \pm}=\left\{\begin{array}{l}n, n \text { integer } \\ \text { largest integer in } n+1, n \text { not integer }\end{array}\right.$

\} if $S_{i}+S_{j}-v<M_{ \pm}$

$N_{ \pm}=\left\{\begin{array}{l}(n-1), n \text { integer } \\ \text { largest integer in } n, n \text { not integer }\end{array}\right.$

\} if $S_{i}+S_{j}-v \geqslant M_{ \pm}$

and where $N_{ \pm}=1$ means non-derivative constraints, $N_{ \pm}=2$ means first derivative constraints etc.

Once $N_{ \pm}$is known the total number of constraints at $\tau_{i j}$ (again for $\lambda_{a}, \lambda_{b}$ fixed but unspecified) $C_{ \pm}$can be found by

$$
C_{ \pm}=N_{ \pm} M_{ \pm}-\left\{\frac{1}{2}\left(N_{ \pm}\right)\left(N_{ \pm}-1\right)\left(1 \leftarrow \alpha_{ \pm}\right)+2\left(N_{ \pm}-1\right)\right\}+\zeta_{i j},
$$

where

$\zeta_{i j}=\left\{\begin{array}{l}1 \text { if } \min \left(M_{ \pm}, S_{i}+S_{i}-v\right)=S_{i}+S_{j}-v \text { and } n \text { not integer and } n>1, \\ 0 \text { otherwise, }\end{array}\right.$

$$
C_{ \pm}=0 \text { whenever the right-hand side of eq. (5) is }<0 \text {. }
$$

The above discussion can be summarized by giving a list of rules for finding all of the kinematical constraints for the process $1+2 \rightarrow 3+4$ at any threshold (pseudothreshold) $\tau_{i j}$ of particles $i, j$ where $a, b$ represent the other two particles (recall that except for $\mathrm{BF} \rightarrow \mathrm{BF}$ we can use the $s$ plane).

(i) Using parity conservation and time reversal invariance determine the total number of independent amplitudes $M_{ \pm}$for $\eta=+$ and $\eta=-$ with $\lambda_{a}, \lambda_{b}$ fixed but arbitrary.

(ii) Find $\alpha_{ \pm}$(i.e., $\alpha$ for $\eta=+$ and - ) using eq. (2).

(iii) Now use $M_{ \pm}$and $\alpha_{ \pm}$together with eq. (4) to determine the number of derivatives, $N_{ \pm}$, which must be considered in order to find all of the constraints.

(iv) Next use $M_{ \pm}, \alpha_{ \pm}, N_{ \pm}$and eq. (5) to find the total number of constraints $C_{ \pm}$that exist at $\tau_{i j}$ for $\lambda_{a}, \lambda_{b}$ fixed but unspecified.

(v) For each independent amplitude expand eq. (1) with $\Sigma_{D}$ including the term $D=0$ only. Solve this set of equations for all non-derivative constraints.

(vi) If $N_{ \pm}=1$ expand eq. (1) for each independent amplitude with $\Sigma_{D}$ including only $D=0$ and 2 . Solve the resultant equations for the first derivative constraints.

(vii) If $N_{ \pm}>2$ expand eq. (1) with $\Sigma_{D}$ now including $D=0,2,4$ and solve for the second derivative constraints.

(viii) Repeat $N_{ \pm}$times this procedure of adding one term to $\Sigma_{D}$ in eq. (1) and then solving for the constraints.

(ix) After having found all of the constraints at $\tau_{i j}$ let $\lambda_{a}, \lambda_{b}$ vary over all their values consistent with parity conservation and time reversal invariance. 
In order to find all of the kinematical constraints repeat steps (i) - (ix) for each threshold and pseudothreshold.

(x) Use eq. (3) to find the constraints at $s=0$ which exist independently of whether or not $m_{1}=m_{2}$ and/or $m_{3}=m_{4}$.

\section{EXAMPLES}

In order to illustrate this method of finding kinematical constraints and to show its simplicity, we shall study the two processes $\rho \pi \rightarrow \mathrm{NN}$ and $\rho \pi \rightarrow \overline{\mathrm{N}} \Delta$.

(A) $\rho \pi \rightarrow N \bar{N}:$ Because this reaction involves $B B \rightarrow F \bar{F}$ we can work in the $s$ plane. Using parity conservation one easily finds the six independent amplitudes: $\bar{f}_{\frac{1}{2} \frac{1}{2} 10}^{ \pm}, \bar{f}_{\frac{1}{2}-\frac{1}{2} 10}^{ \pm}, \bar{f}_{\frac{1}{2} \frac{1}{2} 00}, \bar{f}_{\frac{1}{2}-\frac{1}{2} 00}$

(a) $N_{s}^{\rho, \pi}$ (normal threshold). Steps (i) and (ii) in the above set of rules imply that $M_{+}=1\left(\bar{f}_{\lambda_{\mathrm{N}}^{+} \lambda_{\mathrm{N}} 10}\right), M_{-}=2\left(\bar{f}_{\lambda_{\mathrm{N}} \lambda_{\mathrm{N}}}^{-} 10, \bar{f}_{\lambda_{\mathrm{N}} \lambda_{\mathrm{N}} 00}^{-}\right)$and that $\alpha_{+}=1$, $\alpha_{-}=0$. Then using steps (iii) and (iv) we have $N_{+}=1, N_{-}=1$ and $C_{+}=0$, $C_{-}=1$. Thus we have no constraints for $\eta=+$ and one non-derivative constraint for $\eta=-$. For $\eta=-$ we only need step $(v)$ since $N_{-}=1$. Using eq.

(1) we have

$$
\begin{aligned}
& \tilde{f}_{\lambda_{\mathrm{N}} \lambda_{\mathrm{N}} 00}^{-}=\beta \frac{\langle 1100 \mid 11\rangle}{\sqrt{2}} g_{1}^{0,-}, \\
& \tilde{f}_{\lambda_{\mathrm{N}} \lambda_{\mathrm{N}} 00}=\beta \frac{\langle 1000 \mid 10\rangle}{1} g_{1}^{0,-}, \quad \beta=\mathrm{e}^{-i \pi\left(\lambda_{\rho}-\lambda_{\pi}\right)} .
\end{aligned}
$$

(b) $P_{s}^{\rho, \pi}$ (pseudothreshold). We find the same results as at $N_{s}^{\rho, \pi}$.

(c) $N_{S}^{\mathrm{NN}}$ (normal threshold). Steps (i) and (ii) imply that $M_{+}=2\left(f_{\frac{1}{2}}^{+} \frac{1}{2} \lambda_{\rho} \lambda \pi^{\prime}\right.$, $\left.\bar{f}_{\frac{1}{2}-\frac{1}{2} \lambda_{\rho} \lambda_{\pi}}^{+}\right), M_{-}=2\left(\bar{f}_{\frac{1}{2} \frac{1}{2}}^{-} \lambda_{\rho} \lambda_{\pi}, \bar{f}_{\frac{1}{2}-\frac{1}{2} \lambda_{\rho} \lambda_{\pi}}\right)$ and $\alpha_{+}=0, \alpha_{-}=1$. From (iii) and (iv) $N_{+}=1, N_{-}=1$ and $C_{+}=1, C_{-}=0$. For $\eta=+$ we only need step (v)

$$
\begin{aligned}
& \tilde{f}_{\frac{1}{2} \frac{1}{2} \lambda_{1} \lambda_{2}}^{+}=\beta \frac{\left\langle\frac{1}{2} \frac{1}{2} \frac{1}{2}-\frac{1}{2} \mid 10\right\rangle}{\sqrt{2}} g_{1}^{0,+}, \\
& \tilde{f}_{\frac{1}{2}-\frac{1}{2} \lambda_{1} \lambda_{2}}^{+}=\beta \frac{\left\langle\frac{11 \frac{1}{2} \frac{1}{2}|11\rangle}{2}\right.}{1} g_{1}^{0,+}, \quad \beta=\mathrm{e}^{i \pi\left(\lambda_{\mathrm{N}}-\lambda \overline{\mathrm{N}}\right)} .
\end{aligned}
$$

(d) $P_{S}^{\mathrm{N} \bar{N}}$ (pseudothreshold). We find the same results as at $N_{S}^{\mathrm{N} \bar{N}}$ except $\eta=+(-)$ is replaced by $\eta=-(+)$ everywhere.

(e) $s=0$. Using eq. (3)

$$
-\tilde{f}_{\frac{1}{2}-\frac{1}{2} 10}^{+}=\tilde{f}_{\frac{1}{2}-\frac{1}{2} 10}^{-} .
$$

Therefore we can summarize 
at $N_{S}^{\rho, \pi}$ :

$$
\begin{aligned}
& -\tilde{f}_{\frac{1}{2} \frac{1}{2} 10}^{-}(s)=\frac{1}{\sqrt{2}} \tilde{f}_{\frac{11}{2} \frac{1}{2} 00}^{-}(s), \\
& -\tilde{f}_{\frac{1}{2}-\frac{1}{2} 10}(s)=\frac{1}{\sqrt{2}} \tilde{f}_{\frac{1}{2}-\frac{1}{2} 00}^{-}(s) .
\end{aligned}
$$

at $P_{S}^{p, \pi}$ : same as $N_{S}^{\rho, \pi}$. at $N_{S}^{\mathrm{NN}}$ :

$$
\tilde{f}_{\frac{11}{2} \frac{1}{2} 10}^{+}(s)=\frac{-1}{\sqrt{2}} \tilde{f}_{\frac{1}{2}-\frac{1}{2} 10}^{+}(s) .
$$

at $s=0$ (also $P_{S}^{\mathrm{N}} \overline{\mathrm{N}}$ if $m_{\mathrm{N}}=m_{\overline{\mathrm{N}}}$ ):

$$
\begin{aligned}
& \tilde{f}_{\frac{11}{2} \frac{1}{2} 10}(s)=\frac{(-)}{\sqrt{2}} \tilde{f}_{\frac{1}{2}-\frac{1}{2} 10}^{-}(s)=\frac{1}{\sqrt{2}} \tilde{f}_{\frac{1}{2}-\frac{1}{2} 10}^{+}(s), \\
& \tilde{f}_{\frac{1}{2} \frac{1}{2} 00}^{-}(s)=\frac{(-)}{\sqrt{2}} \tilde{f}_{\frac{1}{2}-\frac{1}{2} 00}^{-}(s) .
\end{aligned}
$$

These results agree with the method of invariant amplitudes [7].

(B) $\rho \pi \rightarrow \Delta \overline{\mathrm{N}}$ : As in example (A) we can use the $s$-plane. In this case we have 12 independent amplitudes $\left(\bar{f}_{\lambda_{\Delta}}^{ \pm} \overline{\mathrm{N}}_{10}, \bar{f}_{\lambda_{\Delta}}^{ \pm} \overline{\mathrm{N}}_{\mathrm{N}} 00, \lambda_{\Delta}=\frac{3}{2}, \frac{1}{2}, \lambda_{\overline{\mathrm{N}}}=\frac{1}{2},-\frac{1}{2}\right)$.

(a) $N_{s}^{\rho, \pi}$ see example (A), part (a).

(b) $P_{S}^{\rho, \pi}$ see example (A), part (b).

(c) At the normal threshold $N_{S}^{\Delta \overline{\mathrm{N}}}$ of $\Delta \overline{\mathrm{N}}$ we have

$$
\begin{array}{llll}
\alpha_{+}=1, & C_{+}^{\Delta \overline{\mathrm{N}}}=2, & N_{+}=1, & M_{+}=4, \\
\alpha_{-}=0, & C_{-}^{\Delta \overline{\mathrm{N}}}=4, & N_{-}=2, & M_{-}=4 .
\end{array}
$$

That is we have for $\eta=+, 4$ independent amplitudes, with two non-derivative constraints and for $\eta=-, 4$ independent amplitudes with four constraints which are a mixture of non-derivative and first derivative constraints. Instead of writing down all of the appropriate equations from eq. (1) we shall simply list a typical example for finding the derivative constraints:

$$
\begin{aligned}
& \tilde{f}_{\frac{3}{2}, \frac{1}{2} \lambda_{1} \lambda_{2}}^{-}=\beta\left[\frac{\left\langle\frac{3}{2}, \frac{3}{2}, \frac{1}{2}-\frac{1}{2} \mid 21\right\rangle}{\sqrt{6}} g_{2}^{0,-}+\left\{\frac{\left\langle\frac{3}{2}, \frac{3}{2}, \frac{1}{2}-\frac{1}{2} \mid 21\right\rangle}{\sqrt{6}} g_{2}^{2,-}+\frac{\left\langle\frac{3}{2}, \frac{3}{2}, \frac{1}{2}-\frac{1}{2} \mid 11\right\rangle}{\sqrt{2}} g_{1}^{2,-}\right\}\right. \\
& \left.\times\left(s-m_{\mathrm{N}}+M_{\Delta}\right)^{2}+\left\{\text { terms of order }\left(s-\left(m_{\mathrm{N}}+M_{\Delta}\right)^{2}\right)^{2}\right\}\right] .
\end{aligned}
$$

(a) At the pseudothreshold $P_{S}^{\Delta \bar{N}}$ of $\Delta \overline{\mathrm{N}}$ we have the same solutions as in (c) except $\eta=+(-)$ is replaced by $\eta=-(+)$.

(e) At $s=0$ we have from eq. (3) 


$$
\begin{aligned}
& \tilde{f}_{\frac{3}{2}, \frac{1}{2} 10}^{+}=+\tilde{f}_{\frac{3}{2}, \frac{1}{2} 10}^{-}, \\
& \tilde{f}_{\frac{3}{2},-\frac{1}{2} 10}^{+}=+\tilde{f}_{\frac{3}{2},-\frac{1}{2} 10}^{-}, \\
& \tilde{f}_{\frac{1}{2}-\frac{1}{2} 10}^{+}=+\tilde{f}_{\frac{3}{2},-\frac{1}{2} 10}^{-}
\end{aligned}
$$

Summarizing:

At $N_{s}^{\rho, \pi}$ :

$$
\begin{gathered}
\tilde{f}_{\frac{3}{2}, \frac{1}{2} 10}^{-}(s)=\frac{1}{\sqrt{2}} \tilde{f}_{\frac{3}{2}, \frac{1}{2} 00}^{-}(s), \\
\tilde{f}_{\frac{1}{2} \frac{1}{2} 10}^{-}(s)=\frac{1}{\sqrt{2}} \tilde{f}_{\frac{1}{2} \frac{1}{2} 00}^{-}(s), \\
\tilde{f}_{\frac{3}{2},-\frac{1}{2} 10}^{-}(s)=\frac{1}{\sqrt{2}} \tilde{f}_{\frac{3}{2},-\frac{1}{2} 00}(s), \\
\tilde{f}_{\frac{1}{2}-\frac{1}{2} 10}^{-}(s)=\frac{1}{\sqrt{2}} \tilde{f}_{\frac{1}{2}-\frac{1}{2} 00}^{-}(s) .
\end{gathered}
$$

At $P_{S}^{0, \pi}$ same results as $N_{S}^{\beta \pi}$.

At $N^{\overline{\mathrm{N}} \Delta}$ (for $\lambda_{\rho}=\left\{\begin{array}{l}1 \\ 0\end{array}\right)$

$-\widetilde{f}_{\frac{3}{2}, \frac{1}{2} \lambda_{\rho} 0}^{-}(s)=\tilde{f}_{\frac{3}{2}-\frac{1}{2} \lambda_{\rho} 0}^{-}(s)=\frac{1}{\sqrt{3}} \tilde{f}_{\frac{1}{2} \frac{1}{2} \lambda_{\rho} 0}^{-}(s)=\frac{-1}{\sqrt{3}} \tilde{f}_{\frac{1}{2}-\frac{1}{2} \lambda_{\rho} 0}^{-}(s)$,

(non-derivative constraint),

$\frac{\sqrt{3}\left(\tilde{f}_{\frac{1}{2} \frac{1}{2}} \lambda_{\rho} 0+\tilde{f}_{\frac{1}{2}-\frac{1}{2} \lambda \rho^{0}}\right)+\left(\tilde{f}_{\frac{3}{2}-\frac{1}{2} \lambda \rho^{0}}+\tilde{f}_{\frac{3}{2}-\frac{1}{2} \lambda}^{-} 0^{0}\right.}{\left[s-\left(m_{\Delta}+m_{N}\right)^{2}\right]}=$ finite,

(first-derivative constraint),

$$
\begin{aligned}
& \tilde{f}_{\frac{3}{2} \frac{1}{2} \lambda \rho^{0}}^{+}=-\frac{1}{2} \tilde{f}_{\frac{3}{2}-\frac{1}{2} \lambda \rho 0}^{-}-\sqrt{\frac{3}{2}} \tilde{f}_{\frac{1}{2} \frac{1}{2} \lambda \rho 0,}^{+} \text {, } \\
& \tilde{f}_{\frac{1}{2}-\frac{1}{2} \lambda \rho^{0}}^{+}=-\sqrt{\frac{3}{4}} \tilde{f}_{\frac{1}{2}-{ }_{2}^{3}}^{+} \lambda_{\rho} 0+\frac{1}{\sqrt{2}} \tilde{f}_{\frac{1}{2} \frac{1}{2} \lambda \rho^{0}}^{+} .
\end{aligned}
$$

At $P_{S}^{\Delta \bar{N}}$ have same results as $N_{S}^{\Delta \mathrm{N}}$ except $\eta=+(-)$ replaced by $\eta=-\left(_{+}\right)$ every where. 
At $s=0$ :

$$
\begin{aligned}
& \tilde{f}_{\frac{3}{2} \frac{1}{2} 10}^{+}(s)=\tilde{f}_{\frac{3}{2} \frac{1}{2} 10}^{-}(s), \\
& \tilde{f}_{\frac{3}{2}-\frac{1}{2} 10}^{+}(s)=\tilde{f}_{\frac{3}{2}-\frac{1}{2} 10}^{-}(s), \\
& \tilde{f}_{\frac{1}{2}-\frac{1}{2} 10}^{+}(s)=\tilde{f}_{\frac{1}{2}-\frac{1}{2} 10}^{-}(s)
\end{aligned}
$$

I would like to thank Dr. F. Henyey for many helpful suggestions and Drs. G. Kane, M. Ross and Y-P. Yao for useful critical evaluations for this paper.

\section{APPENDIX A}

This paper shall be concerned with the process $1+2 \rightarrow 3+4$ where we define (see fig. 1)

$$
\begin{aligned}
& t=\left(P_{3}-P_{1}\right)^{2} \\
& s=\left(P_{1}+P_{2}\right)^{2}=(\text { c.m. energy })^{2}, \\
& u=\left(P_{4}-P_{1}\right)^{2} \text {, } \\
& w=\sqrt{s}, \\
& N_{w^{+}}^{i, j}=w+\left(m_{i}+m_{j}\right), \quad P_{w^{+}}^{i, j}=w+\left(m_{i}-m_{j}\right), \\
& N_{w^{-}}^{i, j}=w-\left(m_{i}+m_{j}\right), \quad P_{w^{-}}^{i, j}=w-\left(m-m_{j}\right), \\
& N_{s}^{i, j}=s-\left(m_{i}+m_{j}\right)^{2} \text { (normal threshold), } i, j=1,2 \text { or } 3,4 \text {, } \\
& P_{s}^{i, j}=s-\left(m_{i}-m_{j}\right)^{2} \text { (pseudothreshold), } \\
& P_{12}=\frac{N_{s}^{1,2} P_{s}^{1,2}}{2 \sqrt{s}}, \quad P_{34}=\frac{N_{t}^{3,4} P_{s}^{3,4}}{2 \sqrt{s}}, \\
& Z \equiv \cos \theta_{s}=\frac{2 s t+s^{2}-s \sum_{1}^{4} m_{i}^{2}+\left(m_{1}^{2}-m_{2}^{2}\right) \cdot\left(m_{3}^{2}-m_{4}^{2}\right)}{N_{s}^{1,2} P_{s}^{1,2} N_{s}^{3,4} P_{s}^{3,4}},
\end{aligned}
$$




$$
\begin{gathered}
\sin \theta_{s}=\frac{2(s \varphi(s, t))^{\frac{1}{2}}}{N_{s}^{1,2} P_{s}^{1,2} N_{s}^{3,4} P_{s}^{3,4}}, \\
\varphi(s, t)=s t\left(\sum_{1}^{4} m_{i}^{2}-s-t\right)-s\left(m_{2}^{2}-m_{4}^{2}\right)\left(m_{1}^{2}-m_{3}^{2}\right)-t\left(m_{1}^{2}-m_{2}^{2}\right)\left(m_{3}^{2}-m_{4}^{2}\right) \\
-\left(m_{1}^{2} m_{4}^{2}-m_{2}^{2} m_{3}^{2}\right)\left(m_{1}^{2}+m_{4}^{2}-m_{2}^{2}-m_{3}^{2}\right),
\end{gathered}
$$

where in order to simplify phase problems we have chosen the same scattering angle as ref. [2] (see fig. 2) and where $\varphi(s, t)=0$ is the physical boundary of the scattering process [8].

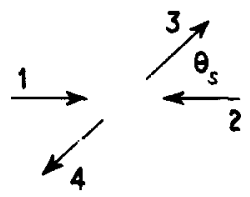

Fig. 2. Phase convention for the process $1+2 \rightarrow 3+4$.

We shall study the problem of kinematical constraints using helicity amplitudes. Since these amplitudes do not possess a definite parity [1], we shall follow the common practice [9] of taking linear combinations of the helicity amplitudes such that the resultant is an amplitude of definite $\eta$ parity $(\eta=(-))^{J} P$ where $J^{-}=$total angular momentum and $P=$ parity). These new amplitudes are called parity conserving helicity amplitudes and are defined by:

$$
\begin{aligned}
\bar{f}_{\lambda_{3} \lambda_{4}, \lambda_{1} \lambda_{2}}^{\eta}(s, t) & =\left(\sqrt{2} \cos \left(\frac{1}{2} \theta\right)\right)^{-|\lambda+\mu|}\left(\sqrt{2} \sin \left(\frac{1}{2} \theta\right)\right)^{-|\lambda-\mu|} f_{\lambda_{3} \lambda_{4}, \lambda_{1} \lambda_{2}} \\
+\eta \eta_{3} \eta_{4}(-1)^{\lambda+\lambda} \max ^{+} & S_{3}+S_{4}-\nu\left(\sqrt{2} \cos \left(\frac{1}{2} \theta\right)\right)^{-|\lambda-\mu|} \\
& \times\left(\sqrt{2} \sin \left(\frac{1}{2} \theta\right)\right)^{-|\lambda+\mu|} f_{-\lambda_{3}-\lambda_{4}, \lambda_{1} \lambda_{2}},
\end{aligned}
$$

where

$$
\begin{array}{ll}
\eta_{i} \equiv \text { intrinsic parity }, & \lambda=\lambda_{1}-\lambda_{2}, \\
S_{i} \equiv \operatorname{spin}, & \mu=\lambda_{3}-\lambda_{4} .
\end{array}
$$

If we assume the usual partial wave expansion for the helicity amplitudes:

$$
f_{\lambda_{3} \lambda_{4}, \lambda_{1} \lambda_{2}}(s, t)=\sum_{0}^{\infty}(2 J+1)\left\langle J M \lambda_{3} \lambda_{4}\right| F^{J}{ }_{\left|J M \lambda_{1} \lambda_{2}\right\rangle d_{\lambda \mu}}^{J}(\theta),
$$


and insert this into eq. (6), we shall find [9] that:

$$
\begin{aligned}
\bar{f}_{\lambda_{3} \lambda_{4}, \lambda_{1} \lambda_{2}}(s, t)=\sum_{0}^{\infty}(2 J+1) e_{\lambda \mu}^{J+}(Z)\left\langle J M \lambda_{3} \lambda_{4}\left|F^{J, \eta}\right| J M \lambda_{1} \lambda_{2}\right\rangle & \\
& +e_{\lambda \mu}^{J-}(Z)\left\langle J M \lambda_{3} \lambda_{4}\left|F^{J(-\eta)}\right| J M \lambda_{1} \lambda_{2}\right\rangle,
\end{aligned}
$$

where, following ref. [9], we have made the following definitions:

$$
\begin{aligned}
& \left\langle J M \lambda_{3} \lambda_{4}\left|F^{J, \eta}\right| J M \lambda_{1} \lambda_{2}\right\rangle=\left\langle\lambda_{3} \lambda_{4}\left|F^{J}\right| \lambda_{1} \lambda_{2}\right\rangle \\
& +\eta \eta_{3} \eta_{4}(-1)^{S_{3}+S_{4}-\nu}\left\langle-\lambda_{3}-\lambda_{4}\left|F^{J}\right| \lambda_{1} \lambda_{2}\right\rangle, \\
& e_{\lambda \mu}^{J \eta}(Z)=\frac{1}{2}\left(\sqrt { 2 } \operatorname { c o s } ( \frac { 1 } { 2 } \theta ) ^ { - | \lambda + \mu | } \left(\sqrt{2} \sin \left(\frac{1}{2} \theta\right)^{-|\lambda-\mu|} d_{\lambda \mu}^{J}(\theta)\right.\right. \\
& +\eta(-1)^{\lambda+\lambda} \max \frac{1}{2}\left[\sqrt{2} \cos \left(\frac{1}{2} \theta\right)\right]^{-|\lambda-\mu|}\left[\sqrt{2} \sin \left(\frac{1}{2} \theta\right)\right]^{-|\lambda+\mu|} d_{\lambda-\mu}^{J}(\theta), \\
& Z \equiv \cos \theta_{s}, \\
& \lambda_{\text {max }}=\max (|\lambda|,|\mu|),
\end{aligned}
$$

with the $J$ and $M$ suppressed on the right-hand side of eq. (8) for convenience.

Finally for later reference we list two useful formulae [9-11]:

$$
\begin{aligned}
& d_{\lambda \mu}^{J}(\theta)=\beta\left\{\frac{\left(J+\lambda_{\max }\right) !\left(J-\lambda_{\max }\right) !}{\left(J+\lambda_{\min }\right) !\left(J-\lambda_{\min }\right) !}\right\}^{\frac{1}{2}}\left[\cos \left(\frac{1}{2} \theta\right)\right]^{b}\left[\sin \left(\frac{1}{2} \theta\right)\right]^{a} P_{J-\lambda \text { max }}^{(a, b)}(Z), \\
& P_{J-\lambda}^{(a, b)}(Z)=\frac{(a+n) !}{(a+b+n) ! n !} \sum_{\alpha=0}^{J-\lambda} \max \frac{(a+b+n+\alpha) !}{2^{\alpha}(a+\alpha) !}\left(\begin{array}{l}
n \\
\alpha
\end{array}\right)(Z-1)^{\alpha}, \\
& a=\left(\lambda_{\max }-\lambda_{\min }\right), \quad \beta=\mathrm{e}^{-i \pi(\lambda-\mu)}, \\
& b=\left(\lambda_{\max }+\lambda_{\min }\right) \text {, } \\
& n=J-\lambda_{\max },
\end{aligned}
$$

where we have followed the phase conventions of ref. [10]. 


\section{APPENDIX B}

\section{Threshold and pseudothreshold constraints}

In this appendix we shall derive eq. (1) which contains the information necessary to determine all of the kinematical constraints at threshold and pseudothreshold for the process $1+2 \rightarrow 3+4$. We shall use the general approach of Jackson and Hite [3] who showed that factorizable kinematical singularities occur because of the mismatch between the zeros of $F^{J}$ and the poles of the $d_{\lambda \mu}^{J}(\theta)$ (see eqs. (10) and (11) which imply that

$$
d_{\lambda \mu}^{J} \approx a_{1} Z^{J-\lambda} \max +a_{2} Z^{J-\lambda} \max ^{-1}+\ldots
$$

where the $a$ are analytic). Jackson and Hite [3] (and Henyey [3] for the case of baryon-fermion scattering) showed that for all masses unequal the scattering amplitude free of factorizable singularities, $\tilde{f} \eta$, can be defined by:

$$
\begin{aligned}
& \tilde{f}_{\lambda_{3} \lambda_{4} ; \lambda_{1} \lambda_{2}}^{\eta}(s, t)=\left\{\left(N_{w^{-}}^{1,2}\right)^{\alpha} N_{w}^{-}\left(N_{w^{+}}^{1,2}\right)^{\alpha} N_{w}^{+}\left(P_{w^{-}}^{1,2}\right)^{\alpha} P_{w}^{-}\left(P_{w^{+}}^{1,2}\right)^{\alpha} P_{w}^{+}\right. \\
& \times\left(N_{w^{-}}^{3,4}\right)^{\beta N_{w}^{-}}\left(N_{w^{+}}^{3,4}\right)^{\beta} N_{w}^{+}\left(P_{w^{+}}^{3,4}\right)^{\beta} P_{w}^{+}\left(P_{w^{-}}^{3,4}\right)^{\beta P_{w}^{-}} \\
&\left.\times s^{\frac{1}{2}(|\lambda|+|\mu|)}\right\}{ }_{\bar{f}_{\lambda 3^{\lambda} 4}^{\eta}, \lambda_{1} \lambda_{2}}^{(s, t),}
\end{aligned}
$$

where

$$
\begin{aligned}
& \alpha_{N_{w}^{-}}=S_{1}+S_{2}-\lambda_{\max }-\frac{1}{2}\left(1-\eta \eta_{1} \eta_{2}(-1)^{S_{1}+S_{2}-\nu}\right), \\
& \alpha_{N_{w}^{+}}^{+}=S_{1}+S_{2}-\lambda \max -\frac{1}{2}\left(1-\eta \eta_{1} \eta_{2}{ }^{(-1)^{-S_{1}-S_{2}-\nu}}\right), \\
& \alpha_{P_{w}^{+}}=S_{1}+S_{2}-\lambda_{\max }-\frac{1}{2}\left(1-\eta \eta_{1} \eta_{2}(-1)^{S_{1}-S_{2}-\nu}\right), \\
& \alpha_{P_{w}^{-}}=S_{1}+S_{2}-\lambda_{\max }-\frac{1}{2}\left(1-\eta \eta_{1} \eta_{2}\left(^{-1)^{-S_{1}+S_{2}-\nu}}\right),\right.
\end{aligned}
$$

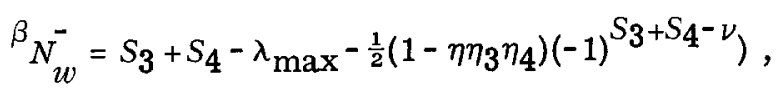

$$
\begin{aligned}
& { }^{\beta} N_{w}^{+}=S_{3}+S_{4}-\lambda \max -\frac{1}{2}\left(1-\eta \eta_{3} \eta_{4}(-1)^{-S_{3}-S_{4}-\nu}\right) \text {, }
\end{aligned}
$$

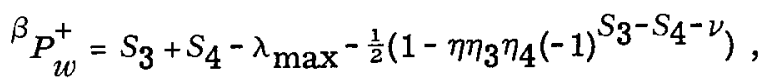

$$
\begin{aligned}
& \beta_{P_{w}^{-}}^{-}=S_{3}+S_{4}-\lambda_{\max }-\frac{1}{2}\left(1-\eta \eta_{3} \eta_{4}(-1)^{-S_{3}+S_{4}-\nu}\right) \text {, }
\end{aligned}
$$

and where 


$$
\begin{aligned}
\nu & =0\left(\frac{1}{2}\right) \quad \text { if } \quad S_{i}+S_{j} \text { integer (half integer), } \\
\lambda_{\max } & =\max (\lambda, \mu), \\
\lambda & =\lambda_{1}-\lambda_{2}, \\
\mu & =\lambda_{3}-\lambda_{4} .
\end{aligned}
$$

For a typical value of $s$ the number on unknowns on the right-hand side of eq. (6) is greater than the number of independent helicity amplitudes and thus no relations exist among the different amplitudes. However at threshold and/or pseudothreshold the existence of kinematical singularities separates the unknowns into groups according to their degree of singularity. Multiplying eq. (6) by the appropriate terms (see eq. (12)) to remove all factorizable kinematical singularities and setting $w$ equal to its values at some threshold which we want to study, say $\left(m_{i}+m_{j}\right)$, only the coefficients of the worst singularities survive (see below). In many cases the number of unknowns now left is less than the number of helicity amplitudes so that we have constraints among the amplitudes (called non-derivative constraints). Repeating this process but dividing by $\left(w-\left(m_{i}+m_{j}\right)\right)^{2}$ (we use the square instead of a linear term because of parity conservation [3]) before setting $w=m_{i}+m_{j}$ we have not only the coefficients of the worst singularities surviving but also the coefficients of the next worst singularities; if the number of unknowns in this case is still less than the number of amplitudes we have first derivative constraints. We can continue this process until the number of unknowns is greater than or equal to the number of amplitudes.

In order to develop this idea more precisely first replace the helicity wave functions of eq. (6) by $L-S$ wave functions [1]:

$$
\begin{gathered}
\left|J M \lambda_{i} \lambda_{j}\right\rangle=\sum_{L, S} \sqrt{\frac{2 L+1}{2 S+1}}\left\langle S_{i} \lambda_{i} S_{j}-\lambda_{j} \mid S_{i j} \lambda_{i}-\lambda_{j}\right\rangle\left\langle S_{i j} \lambda_{i}-\lambda_{j} L 0 \mid J \lambda_{i}-\lambda_{j}\right\rangle|J M L S\rangle, \\
|J M L S\rangle=\sum_{m_{i}, m_{j}}\left\langle S_{i} m_{i} S_{j} m_{j} \mid S_{i j} m_{i}+m_{j}\right\rangle\left\langle S_{i j} m_{i} m_{j} L m \mid J m_{+}+m_{i}+m_{j}\right\rangle\left|L m m_{i} m_{j}\right\rangle, \\
S_{i j}=S_{i}+S_{j}, \quad M=m+m_{i}+m_{j}, \\
m_{i}=\text { projection of } S_{i} \text { onto } Z \text {-axis },
\end{gathered}
$$

which yields:

$$
\begin{gathered}
\bar{f}_{\lambda_{3} \lambda_{4}, \lambda_{1} \lambda_{2}}^{\eta}{ }^{(s, t)}=\sum_{J} \sum_{L_{12}, S_{12}}\left\langle S_{1} \lambda_{1} S_{2}-\lambda_{2} \mid S_{12} \lambda_{1}-\lambda_{2}\right\rangle\left\langle S_{12} \lambda_{1}-\lambda_{2} L_{12} 0 \mid J \lambda_{1}-\lambda_{2}\right\rangle \\
\times \sum_{L_{34}, S_{34}}\left\langle S_{3} \lambda_{3} S_{4}-\lambda_{4} \mid S_{34} \lambda_{3}-\lambda_{4}\right\rangle\left\langle S_{34} \lambda_{3}-\lambda_{4} L_{34} 0 \mid J \lambda_{3}-\lambda_{4}\right\rangle
\end{gathered}
$$


$\times\left\{e_{\lambda \mu}^{J+}(Z) F_{\left(L_{12}, L_{34}, S_{12}, S_{34}, S\right)}^{J \eta}+e_{\lambda \mu}^{J-}(Z) F_{\left(L_{12}, L_{34}, S_{12}, S_{34}, S\right)}^{J(-\eta)}\right.$,

where $F^{J}\left(L_{12}, L_{34}, S_{12}, S_{34}, s\right)$ is shorthand for $\left(\left[\left(2 L_{12}+1\right)\left(2 L_{34}+1\right)\right] /\right.$ $\left.\left[\left(2 S_{12}+1\right)\left(2 S_{34}+1\right)\right]\right)^{\frac{1}{2}} \times\left\langle J M S_{34} L_{34}\left|F^{J}\right| J M S_{12} L_{12}\right\rangle$ and $S_{i j}=S_{i}+S_{j}$.

Next using eqs. (9), (10) and (11) plus the definition [9]

$$
e_{\lambda \mu}(Z)=e_{\lambda \mu}^{J+}(Z)+e_{\lambda \mu}^{J-}(Z)
$$

one can easily derive that:

$$
\begin{aligned}
e_{\lambda \mu}^{J}= & \frac{\frac{(2 J) !}{2 J} \mathrm{e}^{-i \pi(\lambda-\mu)}}{\left(\left(J-\lambda_{\max }\right) !\left(J+\lambda_{\max }\right) !\left(J-\lambda_{\min }\right) !\left(J+\lambda_{\min }\right) !\right)^{\frac{1}{2}}} \\
& \sum_{n=0}^{J-\lambda_{\max }}\left[Z^{J-\lambda} \max ^{-n} \frac{\left(J-\lambda_{\max }\right)\left(J-\lambda_{\max }-1\right) \ldots\left(J-\lambda_{\max }-n+1\right)}{(2 J)(2 J-1) \ldots(2 J-n+1)}\right. \\
& \left.\times \sum_{\eta=0}^{n}\left\{\frac{\prod_{\delta=0}^{\eta=1}\left(J-\lambda_{\min }-\delta\right) \prod_{w=\eta}^{n-1}(2 J-w)}{(n-\eta) ! \eta ! 2^{-\eta}(-1)^{\eta+n}}\right\}\right]
\end{aligned}
$$

where the term in the square brackets is by definition equal to $Z^{J-\lambda} \max$ if $n=0$. And finally, using Racah's equation [12] and a moderate amount of algebra we have for $m$ an integer:

$\langle S \lambda, J-S+m 0 \mid J \lambda\rangle=\left(\frac{m !(2 J-2 S+m) !(2 J+1)(2 S-m) !}{(2 J+m+1) !}\right)^{\frac{1}{2}}$

$\times\left(\frac{(J-S+m) !}{(J-S) !(J-S) !} \frac{(J+\lambda) !(J-\lambda) !}{(S+\lambda) !(S-\lambda) !}\right)^{\frac{1}{2}} \sum_{\nu=0}^{m}\left\{\frac{(-1)^{\nu} \prod_{\zeta=0}^{\nu-1}(S+\lambda-\zeta) \prod_{\gamma=\nu}^{m-1}(S-\lambda-\gamma)}{(m-\nu) ! \prod_{\eta=1}^{m-\nu}(J-S+\eta) \prod_{\beta=1}^{\nu}(J-S+\beta)}\right\}$,

where by assumption

$$
\prod_{n}^{n-a} P(x) \equiv 1, \quad \prod_{n}^{n} P(x) \equiv P(n), \quad a>0 .
$$

For clarity consider explicitly particles $i, j(i, j=1,2$ or 3,4$)$ and substitute eqs. (15) and (16) (with $S=S_{i j}, \lambda=\lambda_{i}-\lambda_{j}$ ) into eq. (4) and collect terms in powers of $\lambda_{i}-\lambda_{j}$ (note that for any $n$ in eq. (15) the possible powers of $\lambda_{\max }$ 
are the same as for $\lambda_{\min }$ so that the expansion form is independent of whether $\left(\lambda_{i}-\lambda_{j}\right)=\lambda_{\max }$ or $\left.\lambda_{\min }\right)$ :

$$
\begin{aligned}
& \bar{f}_{\lambda_{3} \lambda_{4}, \lambda_{1} \lambda_{2}}^{\eta}(s)=\beta \sum_{S_{i j}} \frac{\left\langle S_{i} \lambda_{i} S_{j}-\lambda_{j} \mid S_{i j} \lambda_{i}-\lambda_{j}\right\rangle}{\left(\left(S_{i j}+\lambda_{i}-\lambda_{j}\right) !\left(S_{i j}+\lambda_{j}-\lambda_{i}\right) !\right)^{\frac{1}{2}}} \\
& \quad \times \sum_{n=0}^{J-\lambda_{\max }} \sum_{m=0}^{2\left(S_{i}+S_{j}\right)}\left(\lambda_{i}-\lambda_{j}\right)^{m+n} a^{m, n}\left(\lambda_{a}, \lambda_{b}, S_{a b}, S_{i j}, L_{a b}, J, Z\right),
\end{aligned}
$$

where $a^{m, n}$ is some complicated function of its arguments; $a, b$ refer to the other two particles besides $i, j$; the sum over $m$ goes from zero to $2\left(S_{i}+S_{j}\right)$ because the $\Sigma_{L_{i j}}$ in eq. (14) includes values of $L_{i j}$ from $J-\left(S_{i}+S_{j}\right)$ to $J+\left(S_{i}+S_{j}\right)$; and $\Sigma_{S_{i j}}$ extends only over those values of $S_{i j}$ which can couple [12] to $L=J-\left(S_{i}+S_{j}\right)+m+\alpha$ to give $\boldsymbol{L}+\boldsymbol{S}=\boldsymbol{J}$. For the definition of $\beta$ (see eq. (1). From above we know that eq. (17) has kinematical singularities which can be removed [3] by multiplying by $K(1,2,3,4, w, s)$, where $K(1,2,3,4, w, s)$ is symbolic for the terms in the brackets of eq. (12). Using eqs. (9) - (11) and assuming the usual threshold behavior, $F^{J} \propto P^{L}$ where $P$ is the linear momentum, one can easily see that the scattering amplitude has a zero [3] of order $L_{i j}=J-\left(S_{i}+S_{j}\right)+m+\alpha(m$ positive integer) and a singularity of order $J-\lambda_{\max }-n$ ( $n$ positive integer) and therefore the degree of singularity at threshold (pseudothreshold) is

$$
\begin{aligned}
& \left(J-\lambda_{\max }-n\right)-\left(J-\left(S_{i}+S_{j}\right)+m+\alpha\right)=S_{i}+S_{j}-(D+\alpha)-\lambda_{\max }, \\
& D=m+n, \\
& \alpha=\left\{\begin{array}{l}
0 \\
1
\end{array}\right. \text { depending upon parity conservation (see eq. (3)). }
\end{aligned}
$$

Because for any $D, m=0,1,2, \ldots D$, any value of $S_{i j}$ will contribute to at least one and usually more than one term in the summation of eq. (17) for instance $S_{i j}=S_{i}+S_{j}$ contributes to all terms in eq. (17) while (if $\alpha=0$ )

$S_{i j}=S_{i}+S_{j}-1$ contributes to all terms except for $m=0$ because $S_{i}+S_{j}-1$ cannot couple to $L=J-\left(S_{i}+S_{j}\right)$ to give $J$. Noticing that the coefficients of $a^{m, n}$ in eq. (17) are only functions of $S_{i j}$ for fixed $S_{i}, S_{j}, \lambda_{i}, \lambda_{j}$, we can easily see that for these four quantities fixed and for any fixed $D$ the set of all $a^{m, n}$ with the same value of $S_{i j}$ act effectively as just one unknown function. Therefore eq. (17) becomes:

$$
\begin{array}{r}
\tilde{f}_{\lambda_{3} \lambda_{4}, \lambda_{1} \lambda_{2}}^{\eta}(s)=K(1,2,3,4, w, s) \bar{f}_{\lambda_{3} \lambda_{4}, \lambda_{1} \lambda_{2}}^{\eta}{ }^{(s)} \\
=\beta \sum_{D} \sum_{S_{i j}=S_{i^{+} S_{j}}}^{S_{i^{+} S_{j}-D-\alpha}} \frac{\left\langle S_{i} \lambda_{i} S_{j}-\lambda_{j} \mid S_{i j} \lambda_{i}-\lambda_{j}\right\rangle}{\left(\left(S_{i j}+\lambda_{i}-\lambda_{j}\right) !\left(S_{i j}+\lambda_{j}-\lambda_{i}\right) !\right)^{\frac{1}{2}}}\left(\lambda_{i}-\lambda_{j}\right){ }^{D+\alpha_{\eta}-\xi} \\
\times g_{S_{i j} D, \eta}^{D}\left(\lambda_{a} \lambda_{b} S_{a b} S_{i j} L_{a b} J\right)\left(\sqrt{s}-\tau_{i j}\right)^{\frac{1}{2}}
\end{array}
$$


where $D=0,2,4, \ldots$ because of parity conservation ( $L$ must change by 2), $\Sigma_{S_{i j}}$ is restricted to those values of $S_{i j}$ which can couple to $L=J-\left(S_{i}+S_{j}\right)+$ $+D+\alpha$ to give $L+S=J, g_{S_{i j}}^{D, \eta}$ is the sum of the $a^{m, n}$ of the same $S_{i j}$ mentioned above and $\tau_{i j}$ is symbolic for any one of the four thresholds: $\pm\left(m_{i \pm} m_{j}\right)$. This is eq. (1).

As an aid in using eq. (18) we shall derive an equation for determining the number of 'derivatives' which must be investigated in order to find all constraints (recall that first derivative means including those terms whose singularities are of the second highest degree as well as those of the highest order; second derivative implies including terms whose singularities are of the third, second and highest order, etc.) and we shall also determine the total number of constraints for any two-body scattering process. Recall the important qualification discussed following eq. (2).

As before, consider any threshold or pseudothreshold of particles $i, j$ (we shall not include any of the constraints at $s=0$ which occur independently of whether or not $m_{i}=m_{j}$, i.e., whether or not pseudothreshold is also at $s=0)$. Let $M_{ \pm}$be the number of independent amplitudes for $\eta= \pm$ and $\lambda_{\mathrm{a}}, \lambda_{\mathrm{b}}$ arbitrary but fixed. From eq. (18) we can see that the $D=0$ term introduces $(1+\alpha)$ unknowns since when $D=0$ only $S_{i j}=S_{i}+S_{j}$ or $S_{i}+S_{j}-1$ (if $\alpha \neq 0$ ) can couple to the minimum value of $L$ to give $J$. If $M_{ \pm}>(1+\alpha)$ then there are constraints among the $\tilde{f}_{\lambda_{3} \lambda_{4}, \lambda_{1} \lambda_{2}}$ at $\sqrt{s}=\tau_{i j}$ (non-derivative constraints) because if $\sqrt{s}=\tau_{i j}$ all of the terms but $D=0$ vanish and we then have $M_{ \pm}$equations in $1+\alpha$ unknowns. Since $D=2$ introduces $(1+\alpha+2)$ more unknowns, if $M_{ \pm}>(1+\alpha)+(1+\alpha+2)$ we have more constraints (first derivative constraints) at $\sqrt{s}=\tau_{i j}$ because dividing each $\check{f}_{\lambda_{3} \lambda_{4}, \lambda_{1} \lambda_{2}}(s)$ by $\left(\sqrt{s}=\tau_{i j}\right)$ and then setting $\sqrt{s}=\tau_{i j}$ leaves only the $D=0,2$ terms (there is no trouble with the singularity in the $D=0$ terms because via non-derivative constraints we know how to take combinations of the $\tilde{f}$ to cancel these singular terms). Similarly if $M_{ \pm}>(1+\alpha)+(1+\alpha+2)+(1+\alpha+2)$ we have second derivative constraints, etc. This procedure continues until we have the number of unknowns $\geqslant M_{ \pm}$or until $D>S_{i}+S_{j}$ because then the introduction of larger $D$ does not introduce more unknowns into eq. (18) (i.e., the number of spins in $\Sigma_{S_{i j}}$ cannot become larger). A simple counting procedure will yield eq. (4).

In order to find the total number of constraints add up the number of constraints for each value of $D$ which gives constraints (e.g., if $N_{ \pm}=2$ we have $\left(M_{+}-(1+\alpha)\right)+\left(M_{+}-(1+\alpha+1+\alpha+2)\right)$ constraints). This procedure gives eq. (5). The $\zeta_{i j}$ is added because if for $D=D_{\mathrm{o}}$ the $\Sigma_{S_{i j}}$ in eq. (18) extends from $S_{i j}=S_{i}+S_{j}$ to $\left|S_{i}-S_{j}\right|+1$ and we still have $M_{ \pm}>$number of unknowns (i.e., we have constraints for $D=D_{\mathrm{o}}$ ) and we then let $D$ become $D_{\mathrm{O}}+2$, instead of introducing $1+\alpha+2$ new unknowns we introduce only $1+\alpha+1$ because there is only one new spin which can be included in $\Sigma_{S_{i j}}$; therefore we have one less unknown than is normally true and hence only one extra constraint.

Finally note that eqs. (13), (17), (18) imply that $g_{S_{i j}^{D}, \eta=+}^{D} \neq g_{S_{i j}, \eta=-}^{D}$ at 
at $\sqrt{s}=\tau_{i j}$ because the values of $L_{i j}$ (i.e., $m$ ) differ and therefore we do not expect any constraints between amplitudes of opposite $\eta$ at $\tau_{i j}=\sqrt{s}$.

\section{APPENDIX C}

$s=0$ constraints

In this appendix we derive the constraints which occur at $s=0$ independently of whether or not a pseudothreshold also happens to be at $s=0$. We find that while eq. (1) only gives constraints among amplitudes of the same $\eta$, the $s=0$ constraints are between opposite $\eta$ amplitudes. In order to find these constraints, we use eqs. (8) and (12) to write down the amplitude free of kinematical singularities at $s=0$ for the case of all unequal masses:

$$
\begin{array}{r}
s^{\frac{1}{2}(|\lambda|+|\mu|)} \bar{f}_{\lambda_{3} \lambda_{4}, \lambda}^{\eta} 1_{2}{ }^{(s)}=s^{\frac{1}{2}(|\lambda|+|\mu|)} s^{-\frac{1}{2}(|\lambda-\epsilon \mu|)}(\text { term finite at } s=0) \\
+s^{\frac{1}{2}(|\lambda|+|\mu|)} s^{-\frac{1}{2}(|\lambda+\epsilon \mu|)}(\text { term finite at } s=0), \quad
\end{array}
$$

where $\epsilon= \pm 1$ if $(\cos \theta)_{S=0}= \pm 1$ which is true if $\left(m_{1}^{2}-m_{2}^{2}\right)\left(m_{3}^{2}-m_{4}^{2}\right)>0$ (adopt the convention of choosing positive square roots in definition of $\cos \theta_{S}$ ). If $\lambda$ and $\mu \neq 0$, then either the first or second term is zero depending upon the sign of $\epsilon$ and relative sign of $\lambda, \mu$; therefore $\tilde{f}_{\lambda_{3} \lambda_{4}, \lambda_{1} \lambda_{2}} \propto$ $\propto \tilde{f}_{\lambda_{3} \lambda_{4}, \lambda_{1} \lambda_{2}}^{(-\eta)}$ at $s=0$ for $\lambda, \mu \neq 0$. This result is called a conspiracy because we have a constraint between amplitudes of different quantum numbers. If $\lambda$ and/or $\mu=0$ eq. (21) becomes

$$
\begin{aligned}
s^{\frac{1}{2}(|\lambda|+|\mu|)} \bar{f}_{\lambda_{3} \lambda_{4}, \lambda_{1} \lambda_{2}}^{\eta} & (s)=(\text { finite term at } s=0) \\
& +\eta \text { (finite term at } s=0),
\end{aligned}
$$

which implies that $\bar{f}^{ \pm}$are independent. Using eq. (13) and the fact that at $s=0$ no restrictions are put on $L_{12}, S_{12}, L_{34}, S_{34}$ (other than $L_{12}+S_{12}=$ $=L_{34}+S_{34}=J$ ) one can see that if the $\lambda ' s$ differ for two amplitudes the amplitudes are independent at $s=0$ regardless of whether or not $\lambda$ or $\mu=0$.

We can therefore summarize our discussion of constraints by saying that for each amplitude such that $\lambda, \mu \neq 0$ we have

$$
\begin{aligned}
& \tilde{f}_{\lambda_{3} \lambda_{4}, \lambda_{1} \lambda_{2}}^{+}(s)=-\tilde{a} \frac{\lambda \mu}{|\lambda \mu|} \tilde{f}_{\lambda_{3} \lambda_{4}, \lambda_{1} \lambda_{2}}{ }^{(s)} \\
& \tilde{a}= \pm \text { if }\left(m_{1}^{2}-m_{2}^{2}\right)\left(m_{3}-m_{4}\right) \geq 0 \text { all unequal masses } \\
& = \pm \text { if }\left(m_{a}^{2}-m_{b}^{2}\right)<8, \quad m_{i}=m_{j}, \quad m_{a} \neq m_{b} \\
& =+ \text { if } m_{1}=m_{2}=m_{3}=m_{4} \text {. }
\end{aligned}
$$

This is eq. (3) 


\section{REFERENCES}

[1] M. Jacob and G. Wick, Ann. of Phys. 7 (1959) 404.

[2] L. L. Wang, Phys. Rev. 142 (1966) 1187.

[3] J. Jackson and G. Hite, Phys. Rev. 169 (1968) 1248;

F. Henyey, Phys. Rev. 171 (1968) 1509.

[4] G. Cohen-Tannoudji, A. Morel and H. Navelet, Ann. of Phys. 46 (1968) 239.

[5] D. N. Williams, Lawrence Radiation Laboratory Report URCL-11113, (1963) (unpublished);

G. C. Fox, Phys. Rev. 157 (1967) 1983;

Y. Hara, Phys. Rev. 136 (1964) B507;

H. P. Stapp, Phys. Rev. 160 (1967) 1251;

H. F. Jones, Nuovo Cimento $50 \mathrm{~A}(1967) 814$.

[6] T. L. Trueman, Phys. Rev. 173 (1968) 1684.

[7] B. Diu and M. LeBellac, Nuovo Cimento 50A (1967) 814.

[8] T. Kibble, Phys. Rev. 117 (1959) 1159.

[9] M. Gell-Mann, M. Goldberger, F. Low, E. Marx and F. Zachariasen, Phys. Rev. 133 (1964) B145.

[10] M. Andrews and J. Gunson, J. Math. Phys. 5 (1964) 1391.

[11] Handbook of mathematical functions, ed. by M. Abramowitz and I. A. Stegun (National Bureau of Standards, Applied Mathematics Series, June 1964) p. 771.

[12] D. Brink and G. Satchler, Angular momentum (Oxford University Press, 1962). 